\title{
Interplay between Light and Plant Hormones in the Control of Arabidopsis Seedling Chlorophyll Biosynthesis
}

\author{
Xiaoqin Liu, Yue Li and Shangwei Zhong * \\ State Key Laboratory of Protein and Plant Gene Research, School of Advanced Agricultural Sciences and School of Life \\ Sciences, Peking University, Beijing, China
}

OPEN ACCESS

Edited by:

Chi-Kuang Wen,

Shanghai Institutes for Biological

Sciences (CAS), China

Reviewed by:

Rongcheng Lin,

Institute of Botany (CAS), China

Hao Peng,

Washington State University,

United States

*Correspondence:

Shangwei Zhong

shangwei.zhong@pku.edu.cn

Specialty section:

This article was submitted to

Plant Physiology,

a section of the journal

Frontiers in Plant Science

Received: 30 June 2017 Accepted: 03 August 2017

Published: 17 August 2017

Citation:

Liu X, Li Y and Zhong S (2017) Interplay between Light and Plant

Hormones in the Control

of Arabidopsis Seedling Chlorophyll

Biosynthesis.

Front. Plant Sci. 8:1433.

doi: 10.3389/fpls.2017.01433
Chlorophyll biosynthesis is one of the most important cellular processes and is essential for plant photosynthesis. After germination under the soil, dark-grown seedlings are etiolated and accumulate the chlorophyll precursor protochlorophyllide (Pchlide) in cotyledons. Upon exposure to light, Pchlide is rapidly converted to chlorophyll to initiate photoautotrophic growth. In this light-regulated de-etiolation process, multiple endogenous phytohormones are also involved. Although the co-regulation of seedling greening by light and hormones has long been observed, recent studies greatly advanced our understanding of their interplay by identifying the key components connecting these pathways. The integrators, such as PHYTOCHROME-INTERACTING FACTORs, ELONGATED HYPOCOTYL 5, ETHYLENE INSENSTIVE 3 and DELLA proteins, are key transcription regulators in light or hormone signaling pathways. This review focuses on these integrators and illustrates the regulatory networks of light and hormone interactions in chlorophyll biosynthesis.

Keywords: light signaling, plant hormones, chlorophyll biosynthesis, de-etiolation, PIFs, HY5, EIN3/EIL1, DELLAs

\section{INTRODUCTION}

Crop seeds are usually buried in soil, whereby post-germinative seedlings become etiolated and grow upward heterotrophically from seed reserves. Upon reaching the soil surface, etiolated seedlings undergo a dramatic developmental transition termed de-etiolation, which includes cotyledon opening and greening (Von Arnim and Deng, 1996; Chen et al., 2004). This transition is of particular vulnerability for plant survival, requiring rapid initiation of photoautotrophic growth without causing photooxidation (Huq et al., 2004; Zhong et al., 2014). To successfully accomplish this, chlorophyll biosynthesis must be strictly controlled.

In higher plants, chlorophyll is initially biosynthesized from glutamate, which is then converted to 5-aminolevulinic acid (ALA) and further converted to protochlorophyllide (Pchlide) (Tanaka et al., 2011). When the dark-grown seedlings are exposed to light, the rate-limiting enzymes NADPH protochlorophyllide oxidoreductases (PORs) are photoactivated and catalyze the conversion of Pchlide to chlorophyllide, which is subsequently esterified to mature chlorophyll (Fujita, 1996; Reinbothe et al., 2010). In Arabidopsis, three POR enzymes, PORA, PORB and PORC have been identified, with PORA/PORB playing the main roles in young seedlings (Buhr et al., 2008). Because Pchlide is extremely phototoxic, its amount must be stoichiometrically matched with the level of POR enzymes. Over-accumulation of the free Pchlide that cannot be converted to chlorophyll in time will result in the production of reactive oxygen species (ROS) upon light 
exposure, causing photooxidative damage to the seedlings (op den Camp et al., 2003; Huq et al., 2004; Chen et al., 2013; Zhong et al., 2014). To survive, seedlings have evolved efficient ways to adjust the levels of Pchilde and POR enzymes to allow for rapid establishment of photosynthesis without causing photobleaching (op den Camp et al., 2003; Huq et al., 2004; Zhong et al., 2014). Moreover, carotenoid biosynthesis is also markedly upregulated to protect the etioplasts from photooxidative damage by quenching excess excitation energy when seedlings are exposed to light (Rodriguez-Villalon et al., 2009).

Light is the main environmental factor that regulates the pathway of chlorophyll biosynthesis, while plant hormones are also recruited to mediate the developmental switch of de-etiolation. Some key components in the light signaling pathway, such as PIFs and HY5, connect light signals to the signaling pathways of multiple phytohormones, including ethylene, gibberellin (GA) and cytokinin (CK). In this review, we concentrate on how chlorophyll biosynthesis is cooperatively regulated by light and endogenous hormone signals, focusing on the interplay between light and hormone signaling pathways during seedling de-etiolation.

\section{Chlorophyll Biosynthesis Regulated by Key Components in Light Signaling Pathway}

Light provides plants with energy for photosynthesis and a major source of information about their environment. Both light quality and quantity are constantly monitored by plants through a group of photoreceptors (Quail, 2002; Chen et al., 2004). Among them, phytochromes (phys, including phyA-phyE in Arabidopsis) sense far-red and red light (Quail, 2002; Chen et al., 2004). The perception of light signals by phys initiates an intracellular transduction to alter the expression of nuclear genes (Quail, 2002; Chen et al., 2004; Leivar and Quail, 2011). There are two groups of transcription factors, PIFs and HY5, that mediate light-induced responses in opposite ways (Von Arnim and Deng, 1996; Chen et al., 2004; Leivar and Quail, 2011). PIFs are negative regulators and are directly targeted by photoactivated phys for degradation (Ni et al., 1998, 2014; Huq et al., 2004; Shen et al., 2008), while HY5 is stabilized by light to promote photomorphogenesis (Ang et al., 1998; Osterlund et al., 2000). During the process of de-etiolation, both PIFs and HY5 have been shown to transcriptionally regulate the chlorophyll biosynthesis pathway.

PHYTOCHROME-INTERACTING FACTORs accumulate in dark-grown seedlings and negatively regulate the tetrapyrrole metabolism of chlorophyll biosynthesis (Huq et al., 2004; Monte et al., 2004; Moon et al., 2008; Shin et al., 2009; Stephenson et al., 2009). Mutation of PIF1 and PIF3 results in an excessive amount of Pchlide in the dark and causes severe photobleaching upon light exposure (Huq et al., 2004; Monte et al., 2004; Moon et al., 2008; Shin et al., 2009; Stephenson et al., 2009). Further experiments reveal that PIF1 directly binds to the promoter of PORC, while PIF3 represses the expression of HEMA1, GUN4 and CHLH genes (Moon et al., 2008; Stephenson et al., 2009). HEMA1 is the main glutamyl-tRNA reductase that catalyzes the rate-limiting step for ALA biosynthesis, while GUN4 and CHLH promote the conversion of ALA to the chlorophyll biosynthetic branch (Stephenson and Terry, 2008; Tanaka et al., 2011). In addition, PIF5 has been shown to be involved in the negative regulation of $\mathrm{CHLH}$ gene expression in etiolated seedlings (Shin et al., 2009), and a large portion of nuclear-encoded chlorophyll biosynthesis genes are notably upregulated in the pifQ mutant (lacking PIF1, PIF3, PIF4 and PIF5 genes) (Leivar et al., 2009; Shin et al., 2009). PIF1 was also found to partly repress the transposase-derived transcription factor FHY3/FAR1-activated gene expression of HEMB1 that encodes the ALA dehydratase (Tang et al., 2012), and chromatinremodeling enzyme BRM interacts with PIF1 to modulate PORC expression (Zhang et al., 2017). Moreover, PIFs have been reported to directly repress the gene expression of PSY (phytoene synthase), which is the main rate-determining enzyme of carotenoid biosynthesis (Toledo-Ortiz et al., 2010). When PIFs are degraded by light, carotenoids are rapidly synthesized to coordinate with chlorophyll biosynthesis, thus facilitating the assembly of functional photosynthetic machinery (ToledoOrtiz et al., 2010). Therefore, PIFs play important roles in the fine tuning of tetrapyrrole metabolism, directly or indirectly regulating chlorophyll biosynthesis and photosynthetic genes to optimize the seedling greening process.

ELONGATED HYPOCOTYL 5 functions downstream of the photoreceptors and central repressors in the light signaling pathway to promote seedling photomorphogenesis. In the dark, HY5 is degraded through the COP1/DET1-mediated ubiquitination degradation pathway (Ang et al., 1998; Osterlund et al., 2000). HY5 plays a vital role in the convergence of blue, red and far-red light-signal pathways for regulating the transcription levels of HEMA1 (McCormac and Terry, 2002). Several nuclearencoding photosynthetic and chlorophyll biosynthesis genes, such as CHLH, GUN4, PORC, CAO and CHL27, are the putative targets of HY5 (Lee et al., 2007). Although roots are heterotrophic organs, lots of chlorophyll accumulates in light-grown $\operatorname{det} 1$ and cop1 mutant roots, and HY5 mediates the process of chlorophyll synthesis in roots (Chory and Peto, 1990; Deng et al., 1992; Ang et al., 1998). In addition, a Myb-like transcription factor REVEILLE1 (RVE1) was recently found to act downstream of phyB to modulate chlorophyll biosynthesis by directly activating PORA expression (Xu et al., 2015; Jiang et al., 2016).

\section{Ethylene Is Crucial for Cotyledon Greening and Survival of Seedling Soil Emergence}

Plant hormones are small molecules that mediate a myriad of cellular responses. Many hormones are involved in light-induced seedling greening. One prominent factor affecting chlorophyll biosynthesis is ethylene, which dramatically represses Pchlide accumulation and induces the gene expression of both PORA and PORB in etiolated seedlings (Zhong et al., 2009, 2010, 2014). Thus, ethylene plays a critical role in protecting cotyledons from photooxidative damage when the seedlings are exposed to light. The effects of ethylene are mediated by EIN3/EIL1, the master transcription factors in the ethylene signaling pathway (Chao 
et al., 1997; Guo and Ecker, 2004). EIN3/EIL1 markedly repress the accumulation of Pchlide and directly bind to the promoters of $P O R A$ and $P O R B$ to activate their gene expression (Zhong et al., 2009, 2010, 2014). Genetic studies reveal that EIN3/EIL1 cooperate with PIF1 and act downstream of COP1 in promoting seedling greening (Zhong et al., 2009). The protein levels of EIN3 are enhanced by COP1 but are decreased by light (Zhong et al., 2009; Shi et al., 2016a,b). In addition, overexpressing EIN3 rescues the far-red light-triggered cotyledon greening defects (Zhong et al., 2009).

After germination in soil, the mechanical impedance of soil boosts ethylene production to adjust seedling morphogenesis to enhance the lifting capacity and protect against mechanical injuries (Zhong et al., 2014; Shen et al., 2016; Shi et al., 2016a). EIN3/EIL1 directly activate two independent pathways, an ERF1 pathway to slow down cell elongation and a PIF3 pathway to control Pchlide biosynthesis (Zhong et al., 2012, 2014). These two pathways are coupled to maintain a suitable amount of Pchilde to rapidly initiate photoautotrophic growth without causing photooxidation upon emergence (Zhong et al., 2014). When seedlings penetrate their way toward the surface, the dim light under the soil increases gradually and represses COP1 protein activity (Shi et al., 2016a). COP1 has been found to be the E3 ligase of EBF1 and EBF2, the F-box proteins of the E3 ligases for EIN3 degradation (Shi et al., 2016a). Therefore, COP1 and ethylene mediate the soil-imposed light and mechanical stress signals, respectively, to adjust EIN3 protein levels in response to soil condition changes when seedlings grow upward in the soil (Shi et al., 2016a). Interestingly, EIN3 also promotes the nuclear enrichment of COP1 protein to generate a positive feedback for EIN3 stability regulation (Yu et al., 2013, 2016). At the moment of emergence and reaching sunlight, photoactivated photoreceptor phyB directly interacts with EIN3 and rapidly degrades EIN3 by bringing it to the E3 ligases EBF1 and EBF2 (Shi et al., 2016b). As a result, the repression of photomorphogenesis by EIN3 and ethylene is rapidly lifted to initiate de-etiolation effectively.

\section{Gibberellin Regulates Chlorophyll Biogenesis Partially via the Light Signaling Pathway}

Seedling de-etiolation is also subject to gibberellin (GA) regulation, as inhibiting gibberellin signaling can induce partial photomorphogenesis in the dark (Alabadi et al., 2004, 2008). DELLAs are a subfamily of the GRAS transcriptional regulators and negatively regulate gibberellin signaling to repress GAmediated responses (Jiang and Fu, 2007). Moreover, DELLAs inhibit the transcription activity of PIF3 and PIF4 through direct blocking of the DNA-recognition domain of these factors (de Lucas et al., 2008; Feng et al., 2008). In dark-grown seedlings, DELLAs accumulate and regulate the biosynthetic pathways of both carotenoid and chlorophyll (Cheminant et al., 2011). DELLAs upregulate the expression of genes involved in chlorophyll biosynthesis (CHLH, PORC and CAO) and photosynthesis (LHCB2.2, PSAG and PSAE-1) in a PIFdependent manner (Cheminant et al., 2011). In addition, DELLAs also positively regulate $P O R A$ and $P O R B$ gene expression independently of PIFs and repress ROS-induced photooxidative damage during de-etiolation (Cheminant et al., 2011). However, the regulation of HY5 on gibberellin-mediated chlorophyll biosynthesis seems more moderate than that of PIFs in dark conditions (Cheminant et al., 2011).

\section{Cytokinin Plays an Important Role in Chlorophyll Biosynthesis and Chloroplast Development}

Exogenous cytokinin treatment induces cotyledon expansion and chloroplast partial differentiation (Chory et al., 1994; Vandenbussche et al., 2007). Two GATA family transcription factors, GNC and CGA1/GNL, are induced by cytokinin and regulate the expression of many chloroplast-related genes (Hudson et al., 2011; Chiang et al., 2012). Dark-grown seedlings display small etioplasts with prolamellar bodies in the absence of cytokinin, while large lens-shaped plastids contain some prothylakoid membranes in the presence of cytokinin (Chory et al., 1994). Recent reports indicate that cytokinin mediates the etioplast-to-chloroplast transition by promoting characteristic ultrastructural changes (Cortleven and Schmulling, 2015; Cortleven et al., 2016). Cytokinin signal is perceived by the receptors AHK2 and AHK3 and transduced to B-type ARR transcription factors (Argyros et al., 2008). ARRs directly regulate the expression of genes in chlorophyll biosynthesis and the light harvesting complex, such as HEMA1 and LHCB6 (Cortleven and Schmulling, 2015; Cortleven et al., 2016). As cytokinin has been reported to increase the protein levels of HY5 (Vandenbussche et al., 2007), it is possible that HY5 is a point of convergence between light and cytokinin signaling pathways.

\section{The Function of Other Plant Hormones in Regulating Seedling Greening}

In addition to the well-documented hormones just described, other hormones are also important in regulating seedling greening. Auxin represses HY5 protein accumulation via IAA14 and its regulatory target ARFs in roots (Kobayashi et al., 2012). Moreover, chlorophyll synthesis genes are markedly activated in detached roots via cytokinin but are repressed by auxin (Kobayashi et al., 2017), suggesting that auxin signaling is also involved in the regulation of chlorophyll biosynthesis in the root greening response. However, further analyses are required to elucidate the regulatory network of auxin and light signals in regulating chlorophyll biosynthesis. Brassinosteroid $(\mathrm{BR})$ is known to be involved in the process of de-etiolation. Many chlorophyll biosynthesis genes are upregulated from the microarray data of BR-insensitive bri1-116 seedlings in darkness (Sun et al., 2010). The key transcriptional factor GATA2 has been identified in mediating the crosstalk between BR and light signaling pathways (Luo et al., 2010). Recently, ABI4 was found to activate COP1 expression to repress seedling de-etiolation (Xu et al., 2016). In addition, strigolactones are reported to also be involved in light signaling via regulating the nuclear localization of COP1 (Tsuchiya et al., 2010), and jasmonate inhibits COP1 activity to promote photomorphogenesis (Zheng et al., 2017). However, the signaling pathway of $\mathrm{ABA}$, strigolactones and 


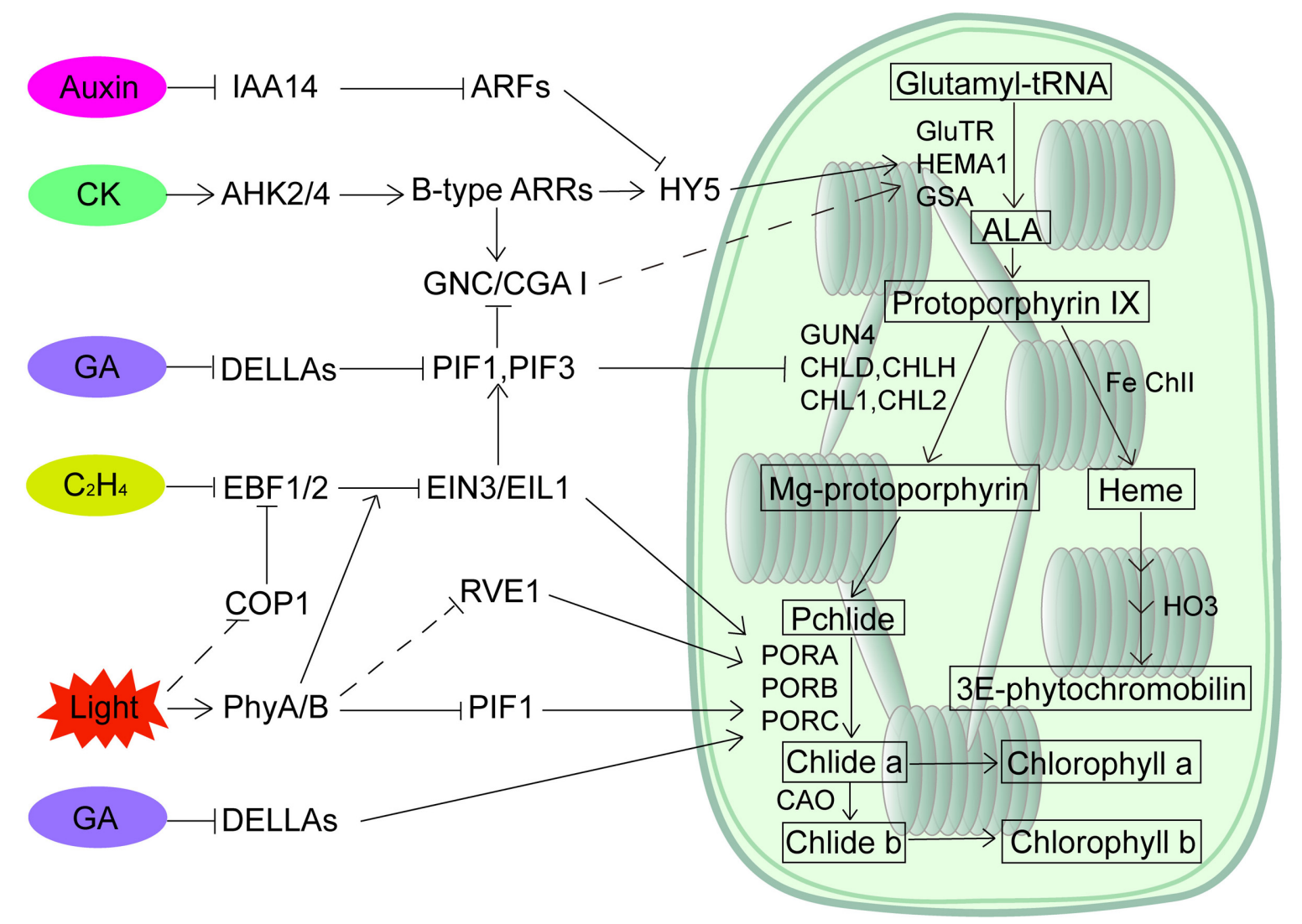

FIGURE 1 | A regulatory network of chlorophyll biosynthesis by light and plant hormones. PIFs and HY5 are major transcription factors in mediating light-regulated chlorophyll biosynthesis. Multiple hormones participate in chlorophyll biosynthesis through both transcriptional and post-transcriptional regulation of PIFs and HY5, while light regulates the action of key components such as EIN3/EIL1 in hormone signaling pathways to modulate the hormonal responses.

jasmonate in regulating chlorophyll biosynthesis remains largely unknown.

\section{CONCLUSION AND PERSPECTIVES}

Involvement of plant hormones in light-regulated seedling greening has been known for decades. However, we have not identified the molecular links connecting light signaling to the multiple hormonal pathways until recent years. The key transcription factors of both light and hormone signaling pathways appear to be the integrators (Figure 1). EIN3 directly activates the gene expression of PORA/PORB and represses Pchlide accumulation to optimize the greening process. The repression of EIN3 in synthesizing Pchlide is through activating PIF3 transcription, whereas both phyB and COP1 predominantly regulate the protein levels of EIN3. PIFs play a pivotal role in integrating light and GA signals, and DELLAs directly sequester the transcription activity of PIFs. In addition, HY5 protein stability is regulated by auxin and cytokinin to coordinate these signals in mediating root greening, while COP1 could be new integrator as its nuclear localization can be regulated by ethylene, strigolactone and jasmonate hormones. Further studies, such as identifying additional integrators in light and hormonal signaling pathways and addressing how these components are integrated in regulating seedling greening, are needed. Moreover, we are only beginning to address the regulation of chloroplast development. Whether and how plant hormones regulate the etioplast-chloroplast differentiation process is critical in filling the gaps of greening. In summary, although we have not obtained a detailed network depicting how seedling greening is regulated by light and all the hormonal signals, the identification of key transcription regulators as signaling integrators has created a great starting point.

\section{AUTHOR CONTRIBUTIONS}

SZ proposed the topic. SZ, XL, and YL collected the literature and critically assessed the information. XL and SZ wrote the manuscript.

\section{FUNDING}

This work was supported by grants from the National Key Research and Development Program of China (2016YFA0502900) and the National Science Foundation of 
China (31570188) to SZ. XL was supported by a China Post-doctoral Science Foundation Grant (2016M600857) and the Outstanding Post-doctoral Fellowship of Peking-Tsinghua Center for Life Sciences.

\section{REFERENCES}

Alabadi, D., Gallego-Bartolome, J., Orlando, L., Garcia-Carcel, L., Rubio, V., Martinez, C., et al. (2008). Gibberellins modulate light signaling pathways to prevent Arabidopsis seedling de-etiolation in darkness. Plant J. 53, 324-335. doi: 10.1111/j.1365-313X.2007.03346.x

Alabadi, D., Gil, J., Blazquez, M. A., and Garcia-Martinez, J. L. (2004). Gibberellins repress photomorphogenesis in darkness. Plant Physiol. 134, 1050-1057. doi: 10.1104/pp.103.035451

Ang, L. H., Chattopadhyay, S., Wei, N., Oyama, T., Okada, K., Batschauer, A., et al. (1998). Molecular interaction between COP1 and HY5 defines a regulatory switch for light control of Arabidopsis development. Mol. Cell 1, 213-222. doi: 10.1016/S1097-2765(00)80022-2

Argyros, R. D., Mathews, D. E., Chiang, Y. H., Palmer, C. M., Thibault, D. M., Etheridge, N., et al. (2008). Type B response regulators of Arabidopsis play key roles in cytokinin signaling and plant development. Plant Cell 20, 2102-2116. doi: 10.1105/tpc.108.059584

Buhr, F., El Bakkouri, M., Valdez, O., Pollmann, S., Lebedev, N., Reinbothe, S., et al. (2008). Photoprotective role of NADPH: protochlorophyllide oxidoreductase A. Proc. Natl. Acad. Sci. U.S.A. 105, 12629-12634. doi: 10.1073/pnas. 0803950105

Chao, Q. M., Rothenberg, M., Solano, R., Roman, G., Terzaghi, W., and Ecker, J. R. (1997). Activation of the ethylene gas response pathway in Arabidopsis by the nuclear protein ETHYLENE-INSENSITIVE3 and related proteins. Cell 89, 1133-1144. doi: 10.1016/S0092-8674(00) 80300-1

Cheminant, S., Wild, M., Bouvier, F., Pelletier, S., Renou, J. P., Erhardt, M., et al. (2011). DELLAs regulate chlorophyll and carotenoid biosynthesis to prevent photooxidative damage during seedling deetiolation in Arabidopsis. Plant Cell 23, 1849-1860. doi: 10.1105/tpc.111.085233

Chen, D., Xu, G., Tang, W., Jing, Y., Ji, Q., Fei, Z., et al. (2013). Antagonistic basic helix-loop-helix/bZIP transcription factors form transcriptional modules that integrate light and reactive oxygen species signaling in Arabidopsis. Plant Cell 25, 1657-1673. doi: 10.1105/tpc.112.104869

Chen, M., Chory, J., and Fankhauser, C. (2004). Light signal transduction in higher plants. Annu. Rev. Genet. 38, 87-117. doi: 10.1146/annurev.genet.38.072902. 092259

Chiang, Y. H., Zubo, Y. O., Tapken, W., Kim, H. J., Lavanway, A. M., Howard, L., et al. (2012). Functional characterization of the GATA transcription factors GNC and CGA1 reveals their key role in chloroplast development, growth, and division in Arabidopsis. Plant Physiol. 160, 332-348. doi: 10.1104/pp.112.198705

Chory, J., and Peto, C. A. (1990). Mutations in the DET1 gene affect cell-typespecific expression of light-regulated genes and chloroplast development in Arabidopsis. Proc. Natl. Acad. Sci. U.S.A. 87, 8776-8780. doi: 10.1073/pnas.87. 22.8776

Chory, J., Reinecke, D., Sim, S., Washburn, T., and Brenner, M. (1994). A role for cytokinins in de-etiolation in Arabidopsis (det mutants have an altered response to cytokinins). Plant Physiol. 104, 339-347. doi: 10.1104/pp.104.2.339

Cortleven, A., Marg, I., Yamburenko, M. V., Schlicke, H., Hill, K., Grimm, B., et al. (2016). Cytokinin regulates the etioplast-chloroplast transition through the two-component signaling system and activation of chloroplast-related genes. Plant Physiol. 172, 464-478. doi: 10.1104/pp.16.00640

Cortleven, A., and Schmulling, T. (2015). Regulation of chloroplast development and function by cytokinin. J. Exp. Bot. 66, 4999-5013. doi: 10.1093/jxb/erv132

de Lucas, M., Daviere, J. M., Rodriguez-Falcon, M., Pontin, M., Iglesias-Pedraz, J. M., Lorrain, S., et al. (2008). A molecular framework for light and gibberellin control of cell elongation. Nature 451, 480-484. doi: 10.1038/nature06520

Deng, X. W., Matsui, M., Wei, N., Wagner, D., Chu, A. M., Feldmann, K. A., et al. (1992). COP1, an Arabidopsis regulatory gene, encodes a protein with both a zinc-binding motif and a G beta homologous domain. Cell 71, 791-801. doi: 10.1016/0092-8674(92)90555-Q

\section{ACKNOWLEDGMENT}

We apologize to our colleagues whose work could not be included because of space constraints.

Feng, S., Martinez, C., Gusmaroli, G., Wang, Y., Zhou, J., Wang, F., et al. (2008). Coordinated regulation of Arabidopsis thaliana development by light and gibberellins. Nature 451, 475-479. doi: 10.1038/nature06448

Fujita, Y. (1996). Protochlorophyllide reduction: a key step in the greening of plants. Plant Cell Physiol. 37, 411-421. doi: 10.1093/oxfordjournals.pcp.a028962

Guo, H., and Ecker, J. R. (2004). The ethylene signaling pathway: new insights. Curr. Opin. Plant Biol. 7, 40-49. doi: 10.1016/j.pbi.2003.11.011

Hudson, D., Guevara, D., Yaish, M. W., Hannam, C., Long, N., Clarke, J. D., et al. (2011). GNC and CGA1 modulate chlorophyll biosynthesis and glutamate synthase (GLU1/Fd-GOGAT) expression in Arabidopsis. PLoS ONE 6:e26765. doi: 10.1371/journal.pone.0026765

Huq, E., Al-Sady, B., Hudson, M., Kim, C., Apel, K., and Quail, P. H. (2004). Phytochrome-interacting factor 1 is a critical bHLH regulator of chlorophyll biosynthesis. Science 305, 1937-1941. doi: 10.1126/science. 1099728

Jiang, C., and Fu, X. (2007). GA action: turning on de-DELLA repressing signaling. Curr. Opin. Plant Biol. 10, 461-465. doi: 10.1016/j.pbi.2007.08.011

Jiang, Z., Xu, G., Jing, Y., Tang, W., and Lin, R. (2016). Phytochrome B and REVEILLE1/2-mediated signalling controls seed dormancy and germination in Arabidopsis. Nat. Commun. 7:12377. doi: 10.1038/ncomms12377

Kobayashi, K., Baba, S., Obayashi, T., Sato, M., Toyooka, K., Keranen, M., et al. (2012). Regulation of root greening by light and auxin/cytokinin signaling in Arabidopsis. Plant Cell 24, 1081-1095. doi: 10.1105/tpc.111.092254

Kobayashi, K., Ohnishi, A., Sasaki, D., Fujii, S., Iwase, A., Sugimoto, K., et al. (2017). Shoot removal induces chloroplast development in roots via cytokinin signaling. Plant Physiol. 173, 2340-2355. doi: 10.1104/pp.16.01368

Lee, J., He, K., Stolc, V., Lee, H., Figueroa, P., Gao, Y., et al. (2007). Analysis of transcription factor HY5 genomic binding sites revealed its hierarchical role in light regulation of development. Plant Cell 19, 731-749. doi: 10.1105/tpc.106. 047688

Leivar, P., and Quail, P. H. (2011). PIFs: pivotal components in a cellular signaling hub. Trends Plant Sci. 16, 19-28. doi: 10.1016/j.tplants.2010.08.003

Leivar, P., Tepperman, J. M., Monte, E., Calderon, R. H., Liu, T. L., and Quail, P. H. (2009). Definition of early transcriptional circuitry involved in lightinduced reversal of PIF-imposed repression of photomorphogenesis in young Arabidopsis seedlings. Plant Cell 21, 3535-3553. doi: 10.1105/tpc.109.070672

Luo, X. M., Lin, W. H., Zhu, S., Zhu, J. Y., Sun, Y., Fan, X. Y., et al. (2010). Integration of light- and brassinosteroid-signaling pathways by a GATA transcription factor in Arabidopsis. Dev. Cell 19, 872-883. doi: 10.1016/j.devcel. 2010.10.023

McCormac, A. C., and Terry, M. J. (2002). Light-signalling pathways leading to the co-ordinated expression of HEMA1 and Lhcb during chloroplast development in Arabidopsis thaliana. Plant J. 32, 549-559. doi: 10.1046/j.1365-313X.2002. 01443.x

Monte, E., Tepperman, J. M., Al-Sady, B., Kaczorowski, K. A., Alonso, J. M., Ecker, J. R., et al. (2004). The phytochrome-interacting transcription factor, PIF3, acts early, selectively, and positively in light-induced chloroplast development. Proc. Natl. Acad. Sci. U.S.A. 101, 16091-16098. doi: 10.1073/pnas.0407107101

Moon, J., Zhu, L., Shen, H., and Huq, E. (2008). PIF1 directly and indirectly regulates chlorophyll biosynthesis to optimize the greening process in Arabidopsis. Proc. Natl. Acad. Sci. U.S.A. 105, 9433-9438. doi: 10.1073/pnas. 0803611105

Ni, M., Tepperman, J. M., and Quail, P. H. (1998). PIF3, a phytochrome-interacting factor necessary for normal photoinduced signal transduction, is a novel basic helix-loop-helix protein. Cell 95, 657-667. doi: 10.1016/S0092-8674(00) 81636-0

Ni, W., Xu, S. L., Tepperman, J. M., Stanley, D. J., Maltby, D. A., Gross, J. D., et al. (2014). A mutually assured destruction mechanism attenuates light signaling in Arabidopsis. Science 344, 1160-1164. doi: 10.1126/science. 1250778

op den Camp, R. G. L., Przybyla, D., Ochsenbein, C., Laloi, C., Kim, C. H., Danon, A., et al. (2003). Rapid induction of distinct stress responses after the 
release of singlet oxygen in arabidopsis. Plant Cell 15, 2320-2332. doi: 10.1105/ tpc. 014662

Osterlund, M. T., Hardtke, C. S., Wei, N., and Deng, X. W. (2000). Targeted destabilization of HY5 during light-regulated development of Arabidopsis. Nature 405, 462-466. doi: 10.1038/35013076

Quail, P. H. (2002). Photosensory perception and signalling in plant cells: new paradigms? Curr. Opin. Cell Biol. 14, 180-188. doi: 10.1016/S0955-0674(02) 00309-5

Reinbothe, C., El Bakkouri, M., Buhr, F., Muraki, N., Nomata, J., Kurisu, G., et al. (2010). Chlorophyll biosynthesis: spotlight on protochlorophyllide reduction. Trends Plant Sci. 15, 614-624. doi: 10.1016/j.tplants.2010.07.002

Rodriguez-Villalon, A., Gas, E., and Rodriguez-Concepcion, M. (2009). Phytoene synthase activity controls the biosynthesis of carotenoids and the supply of their metabolic precursors in dark-grown Arabidopsis seedlings. Plant J. 60, 424-435. doi: 10.1111/j.1365-313X.2009.03966.x

Shen, H., Zhu, L., Castillon, A., Majee, M., Downie, B., and Huq, E. (2008). Light-induced phosphorylation and degradation of the negative regulator PHYTOCHROME-INTERACTING FACTOR1 from Arabidopsis depend upon its direct physical interactions with photoactivated phytochromes. Plant Cell 20, 1586-1602. doi: $10.1105 /$ tpc. 108.060020

Shen, X., Li, Y., Pan, Y., and Zhong, S. (2016). Activation of HLS1 by mechanical stress via ethylene-stabilized EIN3 is crucial for seedling soil emergence. Front. Plant Sci. 7:1571. doi: 10.3389/fpls.2016.01571

Shi, H., Liu, R., Xue, C., Shen, X., Wei, N., Deng, X. W., et al. (2016a). Seedlings transduce the depth and mechanical pressure of covering soil using COP1 and ethylene to regulate EBF1/EBF2 for soil emergence. Curr. Biol. 26, 139-149. doi: 10.1016/j.cub.2015.11.053

Shi, H., Shen, X., Liu, R., Xue, C., Wei, N., Deng, X. W., et al. (2016b). The red light receptor phytochrome B directly enhances substrate-E3 ligase interactions to attenuate ethylene responses. Dev. Cell 39, 597-610. doi: 10.1016/j.devcel.2016. 10.020

Shin, J., Kim, K., Kang, H., Zulfugarov, I. S., Bae, G., Lee, C. H., et al. (2009). Phytochromes promote seedling light responses by inhibiting four negativelyacting phytochrome-interacting factors. Proc. Natl. Acad. Sci. U.S.A. 106, 7660-7665. doi: 10.1073/pnas.0812219106

Stephenson, P. G., Fankhauser, C., and Terry, M. J. (2009). PIF3 is a repressor of chloroplast development. Proc. Natl. Acad. Sci. U.S.A. 106, 7654-7659. doi: 10.1073/pnas.0811684106

Stephenson, P. G., and Terry, M. J. (2008). Light signalling pathways regulating the Mg-chelatase branchpoint of chlorophyll synthesis during de-etiolation in Arabidopsis thaliana. Photochem. Photobiol. Sci. 7, 1243-1252. doi: 10.1039/ b802596g

Sun, Y., Fan, X. Y., Cao, D. M., Tang, W., He, K., Zhu, J. Y., et al. (2010). Integration of brassinosteroid signal transduction with the transcription network for plant growth regulation in Arabidopsis. Dev. Cell 19, 765-777. doi: 10.1016/j.devcel. 2010.10.010

Tanaka, R., Kobayashi, K., and Masuda, T. (2011). Tetrapyrrole metabolism in Arabidopsis thaliana. Arabidopsis Book 9:e0145. doi: 10.1199/tab.0145

Tang, W., Wang, W., Chen, D., Ji, Q., Jing, Y., Wang, H., et al. (2012). Transposase-derived proteins FHY3/FAR1 interact with PHYTOCHROMEINTERACTING FACTOR1 to regulate chlorophyll biosynthesis by modulating HEMB1 during deetiolation in Arabidopsis. Plant Cell 24, 1984-2000. doi: $10.1105 /$ tpc.112.097022

Toledo-Ortiz, G., Huq, E., and Rodriguez-Concepcion, M. (2010). Direct regulation of phytoene synthase gene expression and carotenoid biosynthesis by phytochrome-interacting factors. Proc. Natl. Acad. Sci. U.S.A. 107, 11626-11631. doi: 10.1073/pnas.0914428107
Tsuchiya, Y., Vidaurre, D., Toh, S., Hanada, A., Nambara, E., Kamiya, Y., et al. (2010). A small-molecule screen identifies new functions for the plant hormone strigolactone. Nat. Chem. Biol. 6, 741-749. doi: 10.1038/nchembio.435

Vandenbussche, F., Habricot, Y., Condiff, A. S., Maldiney, R., Van Der Straeten, D., and Ahmad, M. (2007). HY5 is a point of convergence between cryptochrome and cytokinin signalling pathways in Arabidopsis thaliana. Plant J. 49, 428-441. doi: 10.1111/j.1365-313X.2006.02973.x

Von Arnim, A., and Deng, X. W. (1996). Light control of seedling development. Annu. Rev. Plant Physiol. Plant Mol. Biol. 47, 215-243. doi: 10.1146/annurev. arplant.47.1.215

Xu, G., Guo, H., Zhang, D., Chen, D., Jiang, Z., and Lin, R. (2015). REVEILLE1 promotes NADPH: protochlorophyllide oxidoreductase A expression and seedling greening in Arabidopsis. Photosynth. Res. 126, 331-340. doi: 10.1007/ s11120-015-0146-5

Xu, X., Chi, W., Sun, X., Feng, P., Guo, H., Li, J., et al. (2016). Convergence of light and chloroplast signals for de-etiolation through ABI4-HY5 and COP1. Nat. Plants 2:16066. doi: 10.1038/nplants.2016.66

Yu, Y., Wang, J., Shi, H., Gu, J., Dong, J., Deng, X. W., et al. (2016). Salt stress and ethylene antagonistically regulate nucleocytoplasmic partitioning of COP1 to control seed germination. Plant Physiol. 170, 2340-2350. doi: 10.1104/pp.15. 01724

Yu, Y., Wang, J., Zhang, Z., Quan, R., Zhang, H., Deng, X. W., et al. (2013). Ethylene promotes hypocotyl growth and HY5 degradation by enhancing the movement of COP1 to the nucleus in the light. PLoS Genet. 9:e1004025. doi: 10.1371/journal.pgen.1004025

Zhang, D., Li, Y., Zhang, X., Zha, P., and Lin, R. (2017). The SWI2/SNF2 chromatin-remodeling ATPase BRAHMA regulates chlorophyll biosynthesis in Arabidopsis. Mol. Plant 10, 155-167. doi: 10.1016/j.molp.2016.11.003

Zheng, Y., Cui, X., Su, L., Fang, S., Chu, J., Gong, Q., et al. (2017). Jasmonate inhibits COP1 activity to suppress hypocotyl elongation and promote cotyledon opening in etiolated Arabidopsis seedlings. Plant J. 90, 1144-1155. doi: 10.1111/ tpj.13539

Zhong, S., Shi, H., Xi, Y., and Guo, H. (2010). Ethylene is crucial for cotyledon greening and seedling survival during de-etiolation. Plant Signal. Behav. 5, 739-742. doi: $10.4161 /$ psb.5.6.11698

Zhong, S., Shi, H., Xue, C., Wang, L., Xi, Y., Li, J., et al. (2012). A molecular framework of light-controlled phytohormone action in Arabidopsis. Curr. Biol. 22, 1530-1535. doi: 10.1016/j.cub.2012.06.039

Zhong, S., Shi, H., Xue, C., Wei, N., Guo, H., and Deng, X. W. (2014). Ethyleneorchestrated circuitry coordinates a seedling's response to soil cover and etiolated growth. Proc. Natl. Acad. Sci. U.S.A. 111, 3913-3920. doi: 10.1073/ pnas. 1402491111

Zhong, S., Zhao, M., Shi, T., Shi, H., An, F., Zhao, Q., et al. (2009). EIN3/EIL1 cooperate with PIF1 to prevent photo-oxidation and to promote greening of Arabidopsis seedlings. Proc. Natl. Acad. Sci. U.S.A. 106, 21431-21436. doi: $10.1073 /$ pnas. 0907670106

Conflict of Interest Statement: The authors declare that the research was conducted in the absence of any commercial or financial relationships that could be construed as a potential conflict of interest.

Copyright (c) $2017 \mathrm{Liu}, \mathrm{Li}$ and Zhong. This is an open-access article distributed under the terms of the Creative Commons Attribution License (CC BY). The use, distribution or reproduction in other forums is permitted, provided the original author(s) or licensor are credited and that the original publication in this journal is cited, in accordance with accepted academic practice. No use, distribution or reproduction is permitted which does not comply with these terms. 